\title{
Generalized Harmonic Maps and Representations of Discrete Groups
}

\author{
MU-TAO WANG
}

\begin{abstract}
This paper considers generalized harmonic maps from a simplicial complex to a complete metric space of (globally) non-positive curvature. It is proved that if a simplicial complex admits an "admissible weight" satisfying a local combinatorial condition, then any such generalized harmonic maps must be constant maps. The local combinatorial condition is in terms of a nonlinear generalization of the first eigenvalue of a graph. This has applications in the Archimedean and non-Archimedean representations of finitely presentable groups.
\end{abstract}

\section{Introductions.}

Differential geometry is applied to study the representations of finitely presentable discrete groups in this paper. A well known fact is that these groups can be realized as fundamental groups of finite simplicial complexes. The main results here are about the representations of such groups in the isometry group of a complete metric space of (globally) non-positive curvature. This includes all representations in noncompact semisimple real and $p$-adic Lie groups.

The most prominent examples of such discrete groups are lattices in a Lie group $G$. They are discrete subgroups $\Gamma \subset G$ such that the quotients $\Gamma \backslash G$ have finite volumes with respect to the Haar measure on $G$. The rigidity of representations of lattices have been extensively investigated since the early sixties. The theory culminates in Margulis' superrigidity and arithmeticity theorem. Techniques employed by people in studying theses problems include as diverse as representation theory, ergodic theory, linear algebraic group theory and differential geometry.

Along the development, the method of differential geometry has played an important role. The idea is to represent a certain class by a canonical object, and then use the curvature of the symmetric space associated to 
the Lie group to get vanishing theorems. In the local rigidity case, the objects involved are harmonic forms in a certain group cohomology class. While in the global rigidity case the objects involved are harmonic maps in a homotopy class induced by a group representation. Harmonic maps should be viewed as nonlinear versions of harmonic forms.

Though the results proved using differential geometric methods are sometimes not as strong as others, they usually give more intuitive insight into these problems. Actually most early results on group cohomology vanishing theorems and local rigidity theorems are in this direction. Also since differential geometric method only requires local condition, it often applies to some more general category which cannot be approached by methods using global conditions. For example, in the recent proof of local rigidity of hyperbolic cone manifolds by Hodgson and Kerckhoff [8], the universal coverings of such cone manifolds are no longer symmetric spaces and only the local curvature condition is available.

In this article, differential geometric method is applied to study fundamental groups of simplicial complexes. These simplicial complexes are equipped with a weight function on their edges. Analogues of "curvature" are defined on simplicial complexes using the local combinatorial data of the weight function. This can be viewed as a generalization of Garland's $p$-adic curvature in [6]. Also introduced are generalized harmonic maps from simplicial complexes into metric spaces of non-positive curvature. The key point is a nonlinear generalization of Garland's vanishing argument. This technigue was developed in [21] to generalize Margulis' superrigidity for Archimedean representations of lattices in $p$-adic simple Lie groups of rank no less than two. In the present article this argument is extended to treat non-Archimedean representations. The proof involves generalized harmonic maps into Bruhat-Tits buildings, or more generally, complete metric spaces of (globally) non-positive curvature. The local conditions involve the geometry of the tangent cone of these metric spaces.

We remark that the linear case of Garland's argument gives cohomology vanishing theorem which was the content of [6]. It was later elaborated by several groups of authors ( see [1], [22], [17], and [25] ) and applied to proving property $(T)$ of such groups, which is equivalent to the vanishing of all groups cohomology with respect to any irreducible unitary representations in this case.

The ariticle is organized as the following. $\S 2$ contains a brief introduction to rigidity problems and the relations to harmonic maps. $\S 3$ studies the geometry of the tangent cones of a complete metric space of non-positive curvature. The important projection maps from these metric spaces onto 
their tangent cones are also constructed. $\S 4$ defines and proves the existence of generalized harmonic maps from simplicial complexes to complete metric spaces of non-positive curvature. $\S 5$ introduces a notion called the first eigenvalue of a graph in a complete metric space of non-positive curvature. In $\S 6$ the main vanishing theorem is proved . $\S 7$ discusses applications to both Archimedean and non-Archimedean representations.

I am deeply grateful to my advisor Professor Shing-Tung Yau for his guidance and encouragement. Special thanks go to Professor Kazhdan who read a preliminary version of this article and gave several useful suggestions. Part of this work was reported in the colloquium at Yale Univeristy in January, 1998. I would like to thank Professor Garland, Mostow and Margulis for their invitation and interests in this work. In the original version of this work, $\S 3$ and Lemma 6.6 were written only for the case of Bruhat-Tits buildings. It was under the suggestion of the referee that I write up the more general case here. I want to take this chance to thank the referee.

\section{Rigidity problems and harmonic maps.}

We state Mostow's strong rigidity theorem and Margulis' superrigidity theorem in this section and discuss the approach of harmonic maps.

Let $H$ be a real simple noncompact Lie group which is not locally isomorphic to $S L(2, \mathbf{R})$ and $\Gamma \subset H$ a lattice.

Theorem 2.1 (Mostow). If $\rho: \Gamma \mapsto H$ is an injective homomorphism such that $\rho(\Gamma)$ is also a lattice in $H$ then $\rho$ can be extended to be an automorphism of $H$.

Let $X_{1}$ and $X_{2}$ be two complete locally symmetric space associated with $H$ of the same finite volume. In terms of the metric rigidity of locally symmetric spaces, the theorem can be restated as :

Theorem 2.2. An isomorphism between the fundamental groups of $X_{1}$ and $X_{2}$ is induced by an isometry between them.

A natural question is whether there exists a relative version of this picture, i.e. if $G$ is another simple Lie group, can a homomorphism $\rho: \Gamma \mapsto G$ be extended to be a homomorphism $\tilde{\rho}: H \mapsto G$ ? This leads to the superrigidity theorem of Margulis and is true for non-Archimedean simple Lie groups as well. For an Archimedean or non-Archimedean local field $k$, by a $k$-simple Lie group we mean the $k$ rational points of a linear algebraic group which is defined and simple over $k$. 
Theorem 2.3 (Margulis). Let $H$ and $G$ be simple Lie group of Archimedean or non-Archimedean type. Assume $H$ is noncompact and simply connected with rank greater than or equal to two. Let $\Gamma$ be a lattice in $H$. If $\rho: \Gamma \mapsto G$ is Zariski dense then either one of the following is true (1) $\rho$ has bounded image in $G$. (2) $\rho$ extends to a continuous homomorphism $H \mapsto G$, in this case $G$ and $H$ necessarily have the same Archimedean or non-Archimedean type.

The statement of the theorem we depose here includes only the simplest case. Margulis' theorem is true for much more general cases. For the general cases and the proofs of Margulis' superrigidity theorem, we refer to Margulis' [12] or Zimmer's [24] books.

In Margulis' proof, an important step is to show there exists a $\Gamma$ equivariant rational map from $H / H^{\prime}$ to $G / G^{\prime}$, where $H^{\prime} \subset H$ and $G^{\prime} \subset G$ are algebraic parabolic subgroups, see lemma 5.1.3 in [24]. Then such a rational map is shown to extend to a homomorphism from $H$ to $G$.

When $H$ is a real simple Lie group, Margulis's theorem admits a differential geometric proof. The differential geometric method shares similar idea. First we find a $\Gamma$ equivariant harmonic map from $H / H^{\prime \prime}$ to $G / G^{\prime \prime}$, where $H^{\prime \prime} \subset H$ and $G^{\prime \prime} \subset G$ are corresponding maximal compact subgroups. Then use the curvature condition and the Calabi-Matsushima-Weil vanishing argument to show the map is either a constant map or totally geodesic. When this map is totally geodesic, it can be lifted to a homomorphism from $H$ to $G$.

Harmonic maps are generalizations of harmonic functions or harmonic forms. While harmonic functions or harmonic forms can be defined as the critical points of Dirichlet integrals, harmonic maps also allow a variational characterization. Namely, harmonic maps between Riemannian manifolds $M, N$ are critical points $f: M \mapsto N$ of the energy integral

$$
E(f)=\int_{M}\|d f\|^{2} d g_{M}
$$

Here the norm $\|d f\|$ is defined by thinking $d f$ as a section of the bundle $T^{*} M \otimes f^{-1} T N$ with the induced Riemannian metric. Unlike harmonic functions or harmonic forms, harmonic maps satisfy nonlinear partial differential equations.

The application of harmonic maps into rigidity and moduli problems was initiated by Yau. In particular, his idea of using harmonic maps to prove the holomorphic rigidity between quotients of bounded symmetric 
domains was completed by Siu [20]. It was later taken up by Jost-Yau, Mok, and Siu to study rigidity problems on other locally Hermitian symmetric spaces. In 1992, Corlette [5] proved the Archimedean superrigidity for

lattices in $S p(n, 1)$ and $F_{4}^{(-20)}$ using harmonic maps. Later, Gromov and Schoen [7] consider harmonic maps into Bruhat-Tits buildings and prove the non-Archimedean superrigidity for lattices in these groups. These together complete the proof of the arithmeticity of lattices in such real rank 1 groups which was not covered in Margulis' theorem. Their proofs involve a Bochner formula for manifolds with special holonomy. Then Jost-Yau [10] and independently Mok-Siu-Yeung [15] gave the first geometric proof of Margulis' superrigidity theorem for cocompact lattices by elaborating the Calabi-Matsushima-Weil vanishing argument, see [4], [14], and [23].

We try to push this idea to cover more general discrete groups, in particular lattices in $p$-adic simple Lie groups. A key step is to define harmonic maps for general domains with group actions. The model case is the action of $p$-adic lattices on the associated Bruhat-Tits buildings. This leads to our consideration of gereralized harmonic maps on simplicial complexes. Generalized harmonic maps on other domains were also treated in the articles of Jost [9], Korevaar- Schoen [11], and Margulis [13] in different contexts.

\section{Tangent cones of complete metric spaces of non-positive curvature.}

We first recall the definition of complete metric spaces of non-positive curvature.

Definition 3.1. A complete metric space $(N, d)$ is said to be of (globally) non-positive curvature if the following two conditions are satisfied:

(i) $(N, d)$ is a length space. That is, for any two points $P, Q$ the distance is realized as the length of a rectifiable curve connecting $P$ to $Q$. We call such distance realizing curves geodesics.

(ii) For any three points, $P, Q$, and $R$ let $(1-t) Q+t R$ denotes the point on a distance realizing geodesic joining $Q$ and $R$ with distance $t \cdot d(Q, R)$ from $Q$. Then

$$
\begin{aligned}
& d^{2}(P,(1-t) Q+t R) \\
& \leq(1-t) d^{2}(P, Q)+t d^{2}(P, R)-t(1-t) d^{2}(Q, R)
\end{aligned}
$$


Remark 3.3. It is easy to deduce from (ii) that the geodesic joining any two points is unique in $N$.

Our definition of non-positive curvature was borrowed from [11]. There are other definitions of metric spaces of non-positive curvature; for example, Aleksandrov spaces and CAT (0) spaces. Here we require that (ii) holds globally. This condition actually implies $N$ is simply connected at least for locally compact $N$.

We quote a quadrilateral comparison theorem due to Reshetnyak [18] which will be used later.

Theorem 3.4. Let $(N, d)$ be a complete metric space of non-positive curvature. For any $\{P, Q, R, S\}$ ordered four points in $N$, pick $\{\bar{P}, \bar{Q}, \bar{R}, \bar{S}\} \subset \mathbf{R}^{2}$ such that $d(P, Q)=|\bar{P}-\bar{Q}|, d(Q, R)=|\bar{Q}-\bar{R}|, d(R, S)=|\bar{R}-\bar{S}|$, $d(S, P)=|\bar{S}-\bar{P}|$, then for any $0 \leq \lambda, \delta \leq 1$

$$
\begin{aligned}
d((1-\lambda) P+\lambda S,(1-\delta) Q+\delta R) & \\
& \leq|(1-\lambda) \bar{P}+\lambda \bar{S}-(1-\delta) \bar{Q}+\delta \bar{R}|
\end{aligned}
$$

Proof. See [11] Corollary 2.1.2.

A locally compact simply connected complete metric space of nonpositive curvature $N$ allows a compactifition $\bar{N}=N \cup \partial N$ by using the equivalence classes of geodesic rays. Any isometry action induces a continuous action on the boundary. The convergence in the compactified space can be described as the following. Consider any sequence $\left\{a_{i}\right\}$ in $N$, pick up any base point $o$ in $N$. There exist a small geodesic ball $B_{o}$ centered at $o$ which is compact. Let $l_{i}$ be the unique geodesic joining $o$ and $a_{i}, l_{i}$ intersects with the boundary of $B_{o}$ at a unique point $b_{i}$. The sequence $\left\{b_{i}\right\}$ has a convergent subsequence $b_{i_{j}} \rightarrow b$. Then $\left\{a_{i_{j}}\right\}$ converges to the equivalence class represented by the geodesic joining $o$ and $b$.

Two interesting examples of locally compact complete metric spaces of non-positive curvarure are Riemannian symmetric spaces of non-compact type and Bruhat-Tits building.

We proceed to define the tangent cone of $N$ at a point $P$. First, we define the angle between two geodesic rays at $P$. For any two other points $Q, R$, denote $(1-s) P+s Q$ by $Q_{s}$ and $(1-t) P+t R$ by $R_{t}$. 
Lemma 3.6. The expression

$$
\frac{d^{2}\left(P, Q_{s}\right)+d^{2}\left(P, R_{t}\right)-d^{2}\left(Q_{s}, R_{t}\right)}{2 d\left(P, Q_{s}\right) d\left(P, R_{t}\right)}
$$

is bounded between -1 and 1 and non-decreasing as $s, t \rightarrow 0^{+}$

Proof. By the triangle inequality

$$
\begin{aligned}
& \frac{d^{2}\left(P, Q_{s}\right)+d^{2}\left(P, R_{t}\right)-d^{2}\left(Q_{s}, R_{t}\right)}{2 d\left(P, Q_{s}\right) d\left(P, R_{t}\right)} \\
& \leq \frac{d^{2}\left(P, Q_{s}\right)+d^{2}\left(P, R_{t}\right)-\left(d\left(P, Q_{s}\right)-d\left(P, R_{t}\right)\right)^{2}}{2 d\left(P, Q_{s}\right) d\left(P, R_{t}\right)} \\
& \leq 1
\end{aligned}
$$

Similarly, we can prove it is bounded below by -1 . We show that for $s, t \leq 1$

$$
\frac{d^{2}\left(P, Q_{s}\right)+d^{2}\left(P, R_{t}\right)-d^{2}\left(Q_{s}, R_{t}\right)}{2 d\left(P, Q_{s}\right) d\left(P, R_{t}\right)} \geq \frac{d^{2}(P, Q)+d^{2}(P, R)-d^{2}(Q, R)}{2 d(P, Q) d(P, R)}
$$

The above inequality is equivalent to

$$
d^{2}\left(Q_{s}, R_{t}\right) \leq\left(s^{2}-s t\right) d^{2}(P, Q)+\left(t^{2}-s t\right) d^{2}(P, R)+s t d^{2}(Q, R)
$$

The right hand side of (3.8) is exactly the length of $\left|\bar{Q}_{s}-\bar{R}_{t}\right|$ in the comparison triangle $\overline{P Q R}$ in $\mathbf{R}^{2}$. Therefore (3.8) follows from Theorem 3.4. This implies the monotoneness of the expression (3.7) in general.

We define the angle $\theta$ suspended by $Q$ and $R$ at $P$ by taking $\cos \theta$ to be the limit of (3.7) when $s, t \rightarrow 0^{+}$. It is obvious that this angle depends only on the geodesic rays spanned by $Q$ and $R$ and is denoted by $\theta([Q],[R])$.

Lemma 3.9. $d_{P}(Q, R) \equiv d^{2}(P, Q)+d^{2}(P, R)-2 d(P, Q) d(P, R) \cos \theta([Q],[R])$ is a pseudo-distance function on $N$.

Proof. In the definition of $\cos \theta([Q],[R])$ we may take $s=t$, then $d_{P}(Q, R)$ can be written as

$$
\lim _{t \rightarrow 0^{+}} \frac{1}{t} d\left(Q_{t}, R_{t}\right)
$$


The function $\frac{1}{t} d\left(Q_{t}, R_{t}\right)$ is non-increasing as $t \rightarrow 0^{+}$and thus the limit exists as a pseudo-distance.

Now we can define the tangent cone of $N$ at $P \in N$

Definition 3.11. The tangent cone of $N$ at $P,\left(T_{P} N, d_{P}\right)$ is defined to be the metric space $N / \sim$ where $\sim$ is defined by $Q \sim R$ iff $d_{P}(Q, R)=0$. The projection map $N \rightarrow N / \sim$ is denoted by $\pi_{P}$.

Notice that when $N$ is a Riemannian manifold, the map $\pi_{P}: N \rightarrow T_{P} N$ is just the inverse of the exponential map.

The following lemma is clear from the above characterization of $d_{P}$.

Lemma 3.12. $\pi_{P}$ is distance non-increasing and distance preserving in the radial direction, i.e.

$$
\begin{aligned}
& d_{P}\left(\pi_{P}(Q), \pi_{P}(R)\right) \leq d(Q, R) \\
& d_{P}\left(\pi_{P}(P), \pi_{P}(Q)\right)=d(P, Q)
\end{aligned}
$$

Lemma 3.15. The tangent cones of a complete metric space of (globally) non-positive curvature are again complete metric spaces of (globally) nonpositive curvature.

Proof. Take $\lim _{t \rightarrow 0^{+}} \frac{1}{t} d\left(Q_{t}, R_{t}\right)$ as the definition of the metric on the tangent cone. It follows from taking limit of the non-positive curvature condition (3.2).

This lemma holds for a more general situation where only a upper bound of the curvature is assumed, see [16].

Definition 3.16. We say a complete metric space of non-positive curvature $N$ is well approximated by the tangent cone at $P$ if there exist a $\delta$ such that

$$
d^{2}\left(Q_{s}, R\right) \leq d_{P}^{2}\left(\pi_{P}\left(Q_{s}\right), \pi_{P}(R)\right)+r(s)
$$

for any $Q_{s}=(1-s) P+s Q$ with $d\left(P, Q_{s}\right)<\delta$ and $\lim _{s \rightarrow 0^{+}} \frac{r(s)}{s}=0$.

Actually, we have the following Taylor expansion for $d^{2}\left(Q_{s}, R\right)$.

$$
d^{2}\left(Q_{s}, R\right)=d^{2}(P, R)-2 d(P, R) \cos \theta([Q], R) \cdot s+R(s)
$$


with $\lim _{s \rightarrow 0} \frac{R(s)}{s}=0$ and

$$
\cos \theta([Q], R)=\lim _{s \rightarrow 0} \frac{d^{2}\left(P, Q_{s}\right)+d^{2}(P, R)-d^{2}\left(Q_{s}, R\right)}{2 d\left(P, Q_{s}\right) d(P, R)}
$$

A priori $\theta([Q], R)$ depends not only on the direction but also the location of $R$.

On the other hand, for $d_{P}^{2}\left(\pi_{P}\left(Q_{s}\right), \pi_{P}(R)\right)$ we have

$$
d_{P}^{2}\left(\pi\left(Q_{s}\right), \pi(R)\right)=d^{2}(P, R)-2 d(P, R) \cos \theta([Q],[R]) \cdot s+s^{2}
$$

The condition that $N$ is well approximated by the tangent cone implies $\cos \theta([Q], R)=\cos \theta([Q],[R])$.

Both Riemannian manifolds of non-positive sectional curvature and Bruhat-Tits buildings are well approximated by the tangent cone at all points. In the Riemannian case, this can be deduced from the first variation formula of geodesics. In the building case, the tangent cone is actually locally isometric to the building.

\section{Generalized harmonic maps.}

Let $X$ be a finite simplicial complex with counting measure $\mu$ on its vertices and $\pi_{1}(X)$ the fundamental group of $X$. Denote the set of vertices of $X$ by $X(0)$ and the set of all $i$ dimensional simplices of $X$ by $X(i)$. For any vertices $x_{1}, \cdots x_{i+1},\left\langle x_{1}, \cdots x_{i+1}\right\rangle$ denotes the $i$ simpliex spanned by them if it exists.

Definition 4.1. $h$ is said to be a weight function on $X$ if $h$ is a non-negative symmetric function on $X(0) \times X(0)$ such that $h(x, y)>0$ if $\langle x, y\rangle$ is an edge, and $h(x, y)=0$ otherwise.

We may lift $h$ to a function defined on $\tilde{X}(0) \times \tilde{X}(0)$ which is invariant under the diagonal action of $\pi_{1}(X)$. Here $\tilde{X}$ denotes the universal covering of $X$.

Let $N$ be a metric space and $\rho: \pi_{1}(X) \rightarrow I(N)$ a group homomorphism into the isometry group of $N$. Consider the space of $\rho$ equivariant maps from the set of vertices of the universal covering of $X$ to $N$. That is

$$
\Xi_{\rho}=\left\{f: \tilde{X}(0) \mapsto N, f(\gamma x)=\rho(\gamma) f(x) \text { for all } \gamma \in \pi_{1}(X)\right\}
$$

The generalized energy functional and the generalized harmonic map are defined as the followings. 
Definition 4.3. For any $f \in \Xi_{\rho}$, the $h$-energy functional $E_{h}(f)$ is defined to be

$$
E_{h}(f)=\iint h(x, y) d^{2}(f(x), f(y)) d \mu(x) d \mu(y)
$$

where the integral is taken over $\pi_{1}(X) \backslash \tilde{X} \times \tilde{X}$ by the equivariance of $h$ and $f$.

Definition 4.5. $f \in \Xi_{\rho}$ is said to be a generalized $h$-harmonic map if $E_{h}(f)$ attains the minimum of $E_{h}$ in $\Xi_{\rho}$.

In general, we may consider a measure space $(\tilde{X}, \mu)$ and a group $\Gamma$ acting on $\tilde{X}$ preserving $\mu$. The weight function $h$ is then a non-negative function on $\tilde{X} \times \tilde{X}$ which is $\Gamma$ invariant. This is the general case discussed in [9]. We list two other examples here. It is not known whether our vanishing argument can be extended to these interesting examples.

Example 4.6. If $X$ is a Riemannian manifold of dimension $n$, then we can take $h_{\varepsilon}(x, y)=\frac{1}{\varepsilon^{(n+2)}} \chi_{B(x, \varepsilon)}(y)$, where $\chi_{B(x, \varepsilon)}(y)$ is the characteristic function of the geodesic ball $B(x, \varepsilon)$. The energy $E_{h_{\varepsilon}}(f)$ approaches a constant multiple of the usual energy functional $\int|\nabla f|^{2}$ as $\epsilon$ tends to zero. See [9] and [11].

Example 4.7. Let $(X, \mu)$ be a locally compact group with the Haar measure $\mu$. In [13], the author took a strictly positive continuous function $\phi$ on $X$ satisfying some growth condition and let $h(x, y)=\phi\left(x^{-1} y\right)$.

Let $X$ now be a finite simplicial complex with weight function $h$ and $(N, d)$ a complete metric space of non-positive curvature.

Theorem 4.8. Let $N$ be locally compact. If $\rho: \Gamma=\pi_{1}(X) \mapsto I(N)$ is a homomorphism such that the induced action on $\partial N$ does not have any fixed point then the generalized h-harmonic map exists.

Remark 4.9. The assumption on $\rho$ can be weakened. We only have to assume $\rho$ is reductive in the sense of [9] where a more general existence theorem is proved.

Proof. Let $p$ be the number of vertices of $X$. The space of $\rho$ equivariant map $\Xi_{\rho}$ can be identified with $N^{p}$. We think of $E_{h}$ as a function on $N^{p}$ by picking 
a fundamental domain for the action of $\pi_{1}(X)$ on the univeral covering of $X$. We shall prove the functional $E_{h}$ is proper and convex on $N^{p}$, then there exists a minimum point for $E_{h}$. Convexity of $E_{h}$ follows from the fact that $d^{2}$ is a strictly convex function, see Corollary 2.1.3 in [11]. Suppose $E_{h}$ is not proper, then there exists a sequence $\left\{f_{i}\right\} \rightarrow \infty$ in $N^{p}$ with $E_{h}\left(f_{i}\right)<K$, for some constant $K$. In particular, by passing to a subsequence, we may assume there exists a vertex $v$ such that $f_{i}(v)$ converges to a point in $\partial N$. We may well assume $v$ is a vertex of the fundamental domain. Let $d_{\tilde{X}}$ be the simplicial distance on $\tilde{X}(0)$. Suppose the simplicial distance between $v$ and $\gamma(v)$ is realized by a path $v=x_{0}, x_{1} \cdots x_{d}=\gamma(v)$ where $x_{i}$ are all vertices of $\tilde{X}(0)$, then

$$
\begin{aligned}
d_{N}\left(f_{i}(x), f_{i}(\gamma(x))\right) & \leq \sum_{j=0}^{d-1} d_{N}\left(f_{i}\left(x_{j}\right), f_{i}\left(x_{j+1}\right)\right) \leq c_{1} \sum_{j=0}^{d-1} \sqrt{E_{h}\left(f_{i}\right)} \\
& \leq c_{1} \sqrt{K} d_{\tilde{X}}(v, \gamma(v))
\end{aligned}
$$

Here $c_{1}$ is the maximum of $\frac{1}{h(x, y)}$ for all edges $\langle x, y\rangle$. By the above computation, we see that $d_{N}\left(f_{i}(x), \gamma\left(f_{i}(x)\right)\right)$ is independent of $i$, and therefore $f_{i}(x)$ and $\gamma\left(f_{i}(x)\right)$ define the same limit in $\partial N$. Since this is true for all $\gamma \in \Gamma, \lim f_{i}(x)$ is a fixed point in $\partial N$, a contradiction. The theorem is proved.

\section{Local first eigenvalues in a metric space.}

Let $(\mathcal{M}, \nu)$ be a measure space with a finite non-negative measure $\nu$. Let $\Psi^{\prime}$ be a non-negative symmetric function on $\mathcal{M} \times \mathcal{M}$ and we assume $\Psi(v) \equiv$ $\int_{\mathcal{M}} \Psi^{\prime}(v, u) d \nu(u)$ is finite for all $v$.

Let $\left(T, d_{T}\right)$ be a complete metric space of non-positive curvature. Following [11], we define $L^{2}(\mathcal{M}, T)$ to be the set of separable maps $\phi: \mathcal{M} \rightarrow T$ for which the inverse image of open sets are measurable such that

$$
I_{\phi, \Psi}(\cdot)=\int_{\mathcal{M}} \Psi(u) d_{T}^{2}(\cdot, \phi(u)) d \nu(u)
$$

is finite at some point in $T$.

Lemma 5.2. Let $\phi \in L^{2}(\mathcal{M}, X)$, then there exists a unique point $\bar{\phi}_{\Psi}$ in $T$ minimizing $I_{\phi, \Psi}(\cdot)$.

Proof. See Lemma 2.5.1 in [11] 
In our application, $T$ is going to be the tangent cone of a complete metric space of non-positive curvature. $\bar{\phi}_{\Psi}$ is called the weighted center of mass of $\phi$ with respect to $\Psi$.

The following proposition gives more precise information about the behavior of the function $I \equiv I_{\phi, \Psi}$ near $\bar{\phi}_{\Psi}$.

Proposition 5.3. For any $P \in T$,

$$
I(P) \geq I\left(\bar{\phi}_{\Psi}\right)+d^{2}\left(P, \bar{\phi}_{\Psi}\right) \int_{\mathcal{M}} \Psi(u) d \nu(u)
$$

Proof. Parametrize the geodesic joing $\bar{\phi}_{\Psi}$ and $P$ by $\gamma$ such that $\gamma(0)=\bar{\phi}_{\Psi}$ and $\gamma(1)=P$. By (3.2), we have

$$
\begin{aligned}
\int \Psi(u) d_{T}^{2}(\gamma(t), \phi(u)) \leq & (1-t) \int \Psi(u) d_{T}^{2}(\gamma(0), \phi(u)) \\
& +t \int \Psi(u) d_{T}^{2}(\gamma(1), \phi(u)) \\
& -t(1-t) \int \Psi(u) d_{T}^{2}(\gamma(0), \gamma(1))
\end{aligned}
$$

Since

$$
\int \Psi(u) d_{T}^{2}(\gamma(0), \phi(u)) \leq \int \Psi(u) d_{T}^{2}(\gamma(t), \phi(u))
$$

Plug this into the last inequality, the zeroth order term in $t$ are canceled. Divide every term by $t$, we get

$$
\begin{aligned}
& \int \Psi(u) d_{T}^{2}(\gamma(1), \phi(u)) \\
& \geq \int \Psi(u) d_{T}^{2}(\gamma(0), \phi(u))+(1-t) d_{T}^{2}(\gamma(0), \gamma(1)) \int \Psi(u) d \nu(u)
\end{aligned}
$$

Let $t \rightarrow 0$ and the proposition is proved.

Definition 5.5. The first eigenvalue of $\left(\mathcal{M}, \Psi^{\prime}\right)$ in $T$ is defined to be

$$
\lambda_{1}\left(\mathcal{M}, \Psi^{\prime}, T\right)=\min _{\phi} \frac{\frac{1}{2} \iint \Psi^{\prime}(u, v) d_{T}^{2}(\phi(u), \phi(v)) d \nu(u) d \nu(v)}{\int \Psi(u) d_{T}^{2}\left(\bar{\phi}_{\Psi}, \phi(u)\right) d \nu(u)}
$$

where the minimum is taken over all nonconstant $\phi \in L^{2}(\mathcal{M}, T)$. 
When $T$ is an Euclidean space $\mathbf{R}^{n}$, we have

$$
\lambda_{1}\left(\mathcal{M}, \Psi^{\prime}, \mathbf{R}^{n}\right)=\min _{\phi} \frac{\frac{1}{2} \iint \Psi^{\prime}(u, v)|\phi(u)-\phi(v)|^{2} d \nu(u) d \nu(v)}{\int \Psi(u)|\phi(u)|^{2} d \nu(u)}
$$

The minimum is taken over all $\phi$ with $\int_{\mathcal{M}} \Psi(u) \phi(u) d \nu(u)=0$ because we can translate the weighted center of mass $\bar{\phi}_{\Psi}$ to the origin in $\mathbf{R}^{n}$. It is easy to see $\lambda_{1}\left(\mathcal{M}, \Psi^{\prime}, \mathbf{R}^{n}\right)$ is independent of $n$ and we denote it by $\lambda_{1}\left(\mathcal{M}, \Psi^{\prime}\right)$. When $(\mathcal{M}, \nu)$ is the set of vertices of a graph with counting measure and $\Psi^{\prime}$ is the adjacency matrix of the graph, this coincides with the usual definition of first eigenvalue for a graph.

\section{Vanishing theorem for harmonic maps.}

In this section, we are going to deal with a special class of weight functions. For any vertex $x$ in $X$, we recall that $S t(x)$ denotes the subcomplex formed by simplices containing $x$ and $L k(x)$ the union of the faces of $S t(x)$ that do not meet with $x . L k(x)$ is also endowed with a simplicial structure and $L k(x)(0) \subset X(0)$.

Definition 6.1. We say $h$ is an admissible weight function if $h(x, y)=$ $\int_{X} p(x, y, z) d \mu(z)$ for some nonnegative symmetric function $p$ on $X(0) \times$ $X(0) \times X(0)$. We let $(X, h, p)$ denotes such an admissible weight.

For example, if $X$ is a 2-dimensional simplicial complex such that each edge is the face of at least one 2-simplex, then an admissible weight can be obtained by assigning a positive number to each 2 -simplex.

Definition 6.2. If $(X, h, p)$ is an admissible weight, then $p_{x}=p(x, \cdot, \cdot)$ is called the induced weight on the simplicial complex $L k(x)$.

We remark that $p_{x}$ can also be viewed as a function on $X(0) \times X(0)$ which supports on $L k(x)(0) \times L k(x)(0)$.

Let $h_{x}(\cdot)=\int p_{x}(\cdot, y) d \mu(y)=h(x, \cdot)$, then by Definition 5.5 with $(\mathcal{M}, \nu)=L k(x)(0)$ with counting measure, $\Psi^{\prime}=p_{x}, \Psi=h_{x}$ and $T$ a complete metric space of non-positive curvature, we have

$$
\lambda_{1}\left(L k(x)(0), p_{x}, T\right)=\min _{\phi} \frac{\frac{1}{2} \int_{X} \int_{X} p_{x}(y, z) d_{T}^{2}(\phi(y), \phi(z)) d y d z}{\int_{X} h_{x}(y) d_{T}^{2}\left(\bar{\phi}_{h_{x}}, \phi(y)\right) d y}
$$


for all non-constant $\phi \in L^{2}(L k(x)(0), T)$.

When $T$ is an Euclidean space, we define

Definition 6.4. The local first eigenvalue of an admissble weight $(X, h, p)$ is defined to be

$$
\lambda_{1, l o c}(X, p)=\min _{x \in X(0)} \lambda_{1}\left(\operatorname{Lk}(x)(0), p_{x}\right)
$$

We are ready to prove the vanishing theorem. In the rest of this section, $(N, d)$ is a complete metric space of non-positive curvature. $\pi_{q}: N \mapsto T_{q} N$ is the projection map defined in Definition 3.11. We recall that the tangent cone $T_{P} N$ is again a complete metric space of non-positive curvature and $\lambda_{1}\left(L k(x)(0), p_{x}, T_{P} N\right)$ makes sense.

Lemma 6.6. If $f: \tilde{X}(0) \mapsto N$ is a generalized h-harmonic map and $N$ is well approximated by the tangent cone at $f(x)$, then $\pi_{f(x)}(f(x))$ minimizes the function

$$
\int_{X} h(x, y) d_{f(x)}^{2}\left(\cdot, \pi_{f(x)}(f(y))\right) d \mu(y)
$$

in $T_{f(x)} N$ for any $x$.

Proof. We first note that the integral is actually taking over $L k(x)$. Since $f$ is a generalized $h$-harmonic map, $f(x)$ minimize

$$
I(\cdot)=\int_{X} h(x, y) d^{2}(\cdot, f(y)) d \mu(y) .
$$

Consider any variation along a geodesic ray from $f(x)$ to $Q$, by (3.18), we have

$$
-2 \int h(x, y) d(f(x), f(y)) \cos \theta([Q], f(y)) d \mu(y) \cdot s+R(s) \int h(x, y) d \mu(y) \geq 0
$$

for any direction. Since $\lim _{s \rightarrow 0} \frac{R(s)}{s}=0$, we get

$$
\int h(x, y) d(f(x), f(y)) \cos \theta([Q], f(y)) d \mu(y) \leq 0
$$

By the well approximated by the tangent cone assumption,

$$
\cos \theta([Q], f(y))=\cos \theta([Q],[f(y)]),
$$


therefore

$$
\int h(x, y) d\left(\pi_{f(x)}(f(x)), \pi_{f(x)}(f(y))\right) \cos \theta([Q],[f(y)]) d \mu(y) \leq 0
$$

Integrate the Taylor expansion for the function $d_{f(x)}^{2}\left(\cdot, \pi_{f(x)}(f(y))(3.19)\right.$, the lemma is proved.

Theorem 6.10. Let $N$ be a complete metric space of non-positive curvature which is well approximated by the tangent cone at all points. If $(X, h, p)$ is a finite simplicial complex with an admissible weight such that $\lambda_{1}\left(L k(x)(0), p_{x}, T_{P} N\right)>\frac{1}{2}$ for all $x \in X(0)$ and $P \in N$, then any generalized h-harmonic map from $\tilde{X}(0)$ to $N$ is a constant map.

Proof. We first recall for any point $P$ in $N$, the projection map $\pi_{P}: N \mapsto$ $T_{P} N$ is distance nonincreasing and is distance preserving in the radial direction.

Therefore, for any $x \in X(0)$

$$
\begin{aligned}
\frac{1}{2} \iint p(x, y, z) d_{f(x)}^{2} & \left(\pi_{f(x)}(f(y)), \pi_{f(x)}(f(z))\right) d \mu(y) d \mu(z) \\
& \leq \frac{1}{2} \iint p(x, y, z) d^{2}(f(y), f(z)) d \mu(y) d \mu(z)
\end{aligned}
$$

By the previous lemma and the definition of the first eigenvalue (6.3) applying to the map $\pi_{f(x)} \circ f: L k(x)(0) \mapsto T_{f(x)} N$, the left hand side is no less than

$$
\lambda_{1}\left(L k(x)(0), p_{x}, T_{f(x)} N\right) \int h(x, y) d^{2}(f(x), f(y)) d \mu(y)
$$

Since $\lambda_{1}\left(L k(x), p_{x}, T_{f(x)} N\right)>\frac{1}{2}$, we have

$$
\begin{aligned}
& \int h(x, y) d^{2}(f(x), f(y)) d \mu(y) \\
&<\iint p(x, y, z) d^{2}(f(y), f(z)) d \mu(y) d \mu(z)
\end{aligned}
$$

Now we integrate over $X$ with respect to the variable $x$ and by definition $h(y, z)=\int p(x, y, z) d \mu(x)$. We get a contradiction unless $E_{h}(f)=0$, i.e. $f$ is a constant map. 


\section{Applications to representations of discrete groups.}

In this section, $k$ denotes a non-archimedean completion of either an algebraic number field or an algebraic function field in one variable over a finite field.

We recall by Archimedean representations of $\Gamma$ we mean a homomorphism of $\Gamma$ into a real simple Lie group $G$. In this case we consider harmonic maps into the Riemannian symmetric space associated with $G$ and the tangent spaces are Euclidean spaces. Therefore the first eigenvalue is independent of the target space. The next theorem follows from combining Theorem 4.8 and Theorem 6.10. It was also proved in [21].

Theorem 7.1. If $(X, h, p)$ is a finite simplicial complex with an admissible weight such that $\lambda_{1, l o c}(L k(x), p)>\frac{1}{2}$, then the image of any Zariski dense representation of $\pi_{1}(X)$ in a real simple Lie group with trivial center $G$ is precompact in $G$.

It was pointed out by the referee that if the maximal compact subgroup of $G$ is Zariski closed in $G$, then the theorem implies $\pi_{1}(X)$ does not have any Zariski dense representation in $G$.

This theorem, when applies to Bruhat-Tits buildings, gives a new proof of a case of Margulis' superrigidity theorem. We state it as a corollary.

Corollary 7.2. For any integer $l>1$, there is an integer $M$ such that if $k$ has residue field of cardinality at least $M$, if $H$ is a simply connected $k$ simple Lie group of rank $l$, and if $\Gamma$ is a cocompact lattice in $H$, then the image of any Zariski dense representation of $\Gamma$ in a real simple Lie groups with trivial center $G$ is precompact.

We remark that the restriction of the cardinality of the residue field is not necessary in Margulis' theorem. However, we cannot get rid of this assumption at this moment. The proof of Corollary 7.2, also appeared in [21], consists of estimating the lower bound of $\lambda_{1}$ for Bruhat-Tits buildings. The admissible weight $p$ used in this case is equal to $l(l-1) \tilde{p}$ where $l$ is the dimension of the building and $\tilde{p}(\sigma)$ is the number of $l$ dimensional simplices having $\sigma$ as a face. This estimate essentially appeared in [6] where the author showed the vanishing of the first group cohomology for such lattices.

Now we consider non-Archimedean representations. Let $G$ be a simply connected noncompact $k$-simple Lie group and $N$ denotes the Bruhat-Tits building associated to $G$. $T_{q} N$ is a simplicial cone with a complete metric of non-positive curvature. It is isomorphic to the cone over the link of the 
minimum dimensional simplex containing $q$. For material about Bruhat-Tits buildings, we refer to [3] and [2]. The theorem we proved is the following.

Theorem 7.3. Let $(X, h, p)$ be a finite simplicial complex with an admissible weight such that $\lambda_{1}\left(L k(x)(0), p_{x}, T_{q} N\right)>\frac{1}{2}$ for all $x \in X(0)$ and $q \in N$, then the image of any Zariski dense homomorphism $\rho: \pi_{1}(X) \mapsto G$ lies in a bounded subgroup of $G$.

In general, the first eigenvalue $\lambda_{1}$ is hard to compute. In a later paper we give an estimate in the case when $N$ is a tree, which includes all rank 1 buildings. In this case, the tangent cone of $N$ are $n$-pods $P_{n} \simeq \cup_{i=1}^{n}\left(\mathbf{R}^{+} \cup\right.$ $\{0\}) / \sim$ so that the equivalence relation $\sim$ identify the origin of all $\mathbf{R}^{+} \cup\{0\}$. We show that $\frac{2}{3} \lambda_{1}(\mathcal{G}, h) \leq \lambda_{1}\left(\mathcal{G}, h, P_{n}\right) \leq \lambda_{1}(\mathcal{G}, h)$ and prove a fixed point theorem for actions on trees.

For higher dimensional $N$, so far we can only compute

$$
\lambda_{1}\left(\operatorname{Lk}(x)(0), p_{x}, T_{q} N\right)
$$

in the following example. Let $G=P G L\left(3, \mathbf{Q}_{2}\right)$ where $\mathbf{Q}_{2}$ are 2-adic numbers. $N$ is then a 2-dimensional simplicial complex and there are three types of tangent cones. Firstly, if $q$ is an interior point of a 2-simplex, then $T_{q} N$ is an Euclidean two plane. Secondly, if $q$ is an interior point of a 1-simplex, then $T_{q} N$ is the union of three half planes. Lastly, if $q$ is a vertex, then $T_{q} N$ is a cone over the spherical building associated with $P G L(3, \mathbf{Z} / 2 \mathbf{Z})$. The spherical building associated with $P G L(3, \mathbf{Z} / 2 \mathbf{Z})$ is a bipartite graph with 7 vertices representing points in the projective plane $\mathbf{P}^{2}(\mathbf{Z} / 2 \mathbf{Z})$ and other 7 vertices representing lines in the same space. The edges of this graph is given by the incidence relations in $\mathbf{P}^{2}(\mathbf{Z} / 2 \mathbf{Z})$.

Now we take a torsion-free cocompact lattice $\Gamma$ in $G$ and let $X=\Gamma \backslash N$ be the quotient simplicial complex. Take $p$ so that $p(x, y, z)=1$ if $\langle x, y, z\rangle$ is a 2-simplex and $p(x, y, z)=0$ otherwise. Then $\lambda_{1}\left(\operatorname{Lk}(x)(0), p_{x}, T_{q} N\right)=\frac{1}{2}$ when $q$ is a vertex. This $\lambda_{1}$ is attained by a map $\phi: L k(x)(0) \rightarrow T_{q} N$ so that $d(\phi(x), \phi(y))$ is a constant if $\langle x, y\rangle$ is an edge. It looks very plausibe that in this case we can prove the harmonic map is actually a simplicial isometry.

\section{References.}

[1] W. Ballmann and J. Swiatkowski, On $L^{2}$-cohomology and property (T) for automorphism groups of polyhedral cell complexes, GAFA, 7 (1997), 615-645.

[2] K. Brown, Buildings, Springer-Verlag, New York 1989. 
[3] F. Bruhat J. and Tits, Groupes réductifs sur un corps local. I. Données radicielles valuées, Inst. Hautes Études Sci. Publ. Math. 41 (1972), 5-251.

[4] E. Calabi and E. Vesentini, On compact, locally symmetric Kahler manifolds, Ann. Math. 71 (1960), 472-507.

[5] K. Corlette, Archimedean superrigidity and hyperbolic geometry, Ann. Math. 135 (1992), 165-182.

[6] H. Garland, p-adic curvature and the cohomology of discrete subgroups of $p$ adic groups, Ann. Math. 97 (1973), 375-423.

[7] M. Gromov and R. Schoen, Harmonic maps into singular spaces and p-adic superrigidity for lattices in groups of rank one, Publ. I.H.E.S. 76 (1992), 165246.

[8] C. Hodgson and S. Kerckhoff, Rigidity of hyperbolic cone-manifolds and hyperbolic Dehn surgery, J. Differential Geom. 48 (1998), no. 1, 1-59.

[9] J. Jost, Generalized harmonic maps between metric spaces, in Geometric Analysis and the Calculus of Variations for Stefan Hildebrandt, edited by J. Jost, International Press, 1996.

[10] J. Jost and S-T. Yau, Harmonic maps and superrigidity, Proceedings of Symposia in Pure Mathemaitcs, 54 (1993) Part I, AMS, 245-280.

[11] N. Korevaar and R. Schoen, Sobolev spaces and harmonic maps for metric space target, Comm. Anal. Geom, 1 (1993), 561-659.

[12] G.A. Margulis, Discrete subgroups of semisimple Lie groups, Springer-Verlag, 1991.

[13] G.A. Margulis, Superrigidity for commensurability subgroups and generalized harmonic maps, preprint.

[14] Y. Matsushima, On the first Betti number of compact quotient spaces of higher dimensional symmetric spaces, Ann. of Math. 75 (1962), 312-330.

[15] N. Mok, Y.T. Siu, S.K. and Yeung, Geometric superrigidity , Invent. Math., 113 (1993), 57-83.

[16] I. Nikolaev, The tangent cone of an Aleksandrov space of curvature $\leq K$, Manuscripta Mathematica, 86 (1995), 137-147.

[17] P. Pansu, Formule de Matsushima, de Garland, et propriete (T) pour des groupes agissant sur des espaces symetriques ou des immeubles, preprint (Orsay 1996). 
Generalized harmonic maps and representations of discrete groups $\quad 563$

[18] Y.G. Reshetnyak, Nonexpanding maps in a space of curvature no greater than $K$, Siberian Math. Journ. 9 (1968), 918-927.

[19] M. Ronan, Lectures on Buildings, Academic Press, San Diego 1989.

[20] Y.T. Siu, The complex analyticity of harmonic maps and the strong rigidity of compact Kähler manifolds, Ann. Math. 112 (1980), 73-111.

[21] M-T. Wang, A fixed point theorem of discrete group actions on Riemannian manifolds, J. Differential Geom 50 (1998), no.2, 247-267.

[22] M-T. Wang, Fixed point theorems and property (T), preprint, 1998.

[23] A. Weil, Discrete subgroups of Lie groups II, Ann. Math. 75 (1962), 578-602.

[24] R. Zimmer, Ergodic theory and semisimple groups, Birkhaüser, 1984.

[25] A. Zuk, La propriete (T) de Kazhdan pour les groupes agissant sur les polyedres, C. R. Acad. Sci. Paris 323, Serie I (1996), 453-458.

HARVARD UNIVERSITY

CAMBridge, MA 02138

USA

E-mail address: mtwang@math.harvard.edu

ReCeived April 29, 1998 AND ReVised September 17, 1998. 